\title{
PERFORATED GANGRENE UTERUS: A Case Report
}

\begin{tabular}{ll}
$\begin{array}{l}\text { Suryadevara } \\
\text { Snigdha* }\end{array}$ & Post Graduate, Kims \& Rf, Amalapuram. *Corresponding Author \\
\hline Menda Jhansi & Post Graduate, Kims \& Rf, Amalapuram. \\
\hline $\begin{array}{l}\text { Asha Nirmala } \\
\text { Sabbella }\end{array}$ & Post Graduate, Kims \& Rf, Amalapuram. \\
\hline
\end{tabular}

ABSTRACT

Perforated gangrene uterus is a rare condition with high mortality rate, which is difficult and unable to diagnose in first instance without specific history. A 48 year old woman P3L3 with normal vaginal deliveries, not tubectomised came with clinical features of perforation with peritonitis and underwent emergency exploratory laparotomy and found perforated gangrene uterus. Total Abdominal Hysterectomy with Salpingectomy done and specimen shows necrosis of the tissue. Awareness of many etiologies for peritonitis is of great importance when a fatal infection may be the cause of the condition. The most common cause of gas gangrene is clostridium perfringens.

\section{KEYWORDS : Gas Gangrene, Peritonitis, Clostridium Perfringens, Perforation}

\section{INTRODUCTION:}

Perforated gangrenous uterus is a rare condition with high mortality rate, which is difficult and unable to diagnose in first instance without specific history. Intra-abdominal Clostridium Perfringens gas gangrene infection is extremely rate. It is associated with uterine malignancy and should be considered in gynaecologic patients in whom a common etiology cannot be diagnosed. Prompt and aggressive treatment is essential because of the severity of this infection. This article presents the case of a 48 year old women with sepsis.

\section{CASE REPORT:}

A 48 year woman Para3 Living3 with all normal vaginal deliveries, not tubectomised came with complaining of abdominal pain and fever for 4 days and constipation for 3 days to causality. Initial vitals obtained in the emergency department were as follows- Temperature- 100F, Blood pressure- $130 / 80 \mathrm{mmHg}$, Heart rate- 104bpm, Respiratory rate20 per minute, saturation- $98 \%$ at room air. She was in severe distress because of her abdominal pain. On physical examination revealed that she had a tympanic, distended abdomen that was tender to palpation and that had rebound tenderness, generalised guarding present. History of taking anti-inflammatory drugs present. On investigations, Complete blood count reveal moderate leucocytosis $(13,000 / \mathrm{mm} 3)$ and creatinine level of $2.6 \mathrm{mg} / \mathrm{dL}$ and she was catheterized with Foley Catheter and her urine output was minimal, and Erect X-ray abdomen showing Bilateral gas under the diaphragm, which leads the general surgeon to diagnosis of Acute perforation with peritonitis because woman did not given any interfering history. Patient was taken to operating room for emergency exploratory laparotomy. Upon entry into the abdominal cavity, there was a burst of foul smelling gas. They did not found any duodenal or intestine or stomach perforation. There was $2.5 \mathrm{cms}$ spontaneous rupture of the body of uterus anteriorly. An intrauterine malignancy was suspected and a subsequent emergency Total Abdominal Hysterectomy and salpingectomy were performed and specimen sent for histopathological examination. The peritoneal cavity was copiously irrigated with Normal saline and an antibiotic solution.

Blood gram stain showed large gram positive rods and presence of Clostridium Perfringens was confirmed by blood and intra operative wound cultures. On POD-0 patient was severely academic and was found to be hypotensive. Norepinephrine was administered for pressure support and started antibiotics, piperacillin, tazobactum and metronidazole intravenously. On POD-1, blood and peritoneal fluid cultures returned were positive for Clostridium perfringens and her antibiotic regimen was subsequently changed to vancomycin and intravenous pencillin G. On POD-3 patient expired due to shock following

Sepsis and multi organ failure. We have to rule out while taking history of amenorrhoea. Interference by somebody to abort.

\section{DISCUSSION:}

Till date only 18 cases of uterine infection with gas gangrene have been reported and out of these only 6 cases have been reported in non-pregnant uterus. The most common cause of gas gangrene is clostridium species of this clostridium perfringens being the most common. Clostridium perfringens is an encapsulated gram positive anaerobic, non-motile bacilli and capable of producing exotoxins. Therefore necrotic and injured tissue permits the growth of bacterial colonies and production of toxins. That is why this infection is more commonly seen after deliveries, abortions, molar pregnancies and in patients with underlying malignancies.

\section{Diagnosis:}

It is diagnosed on the basis of blood and wound cultures showing isolation of Clostridium Perfringens organism and Erect X-ray Abdomen showing bilateral gas under diaphragm. Histopathological examination revealing necrosis of the tissue.

\section{CONCLUSION:}

Awareness of many etiologies for peritonitis is of great importance when a fatal infection may be the cause of the condition. Correct diagnosis and proper treatment is essential for the survival of the patients infected.

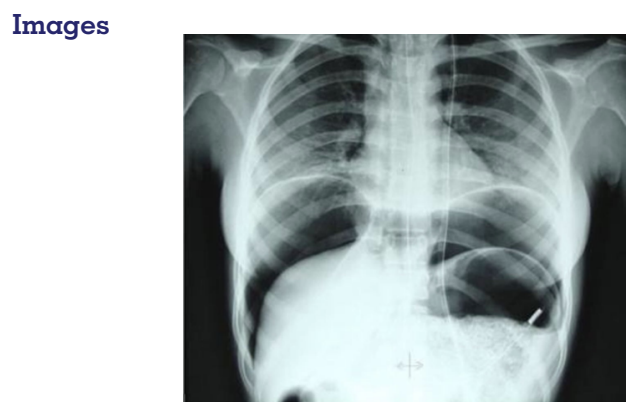

X-ray Erect Abdomen Showing Large Amount Of Air Under Both Domes Of Diaphragm. 


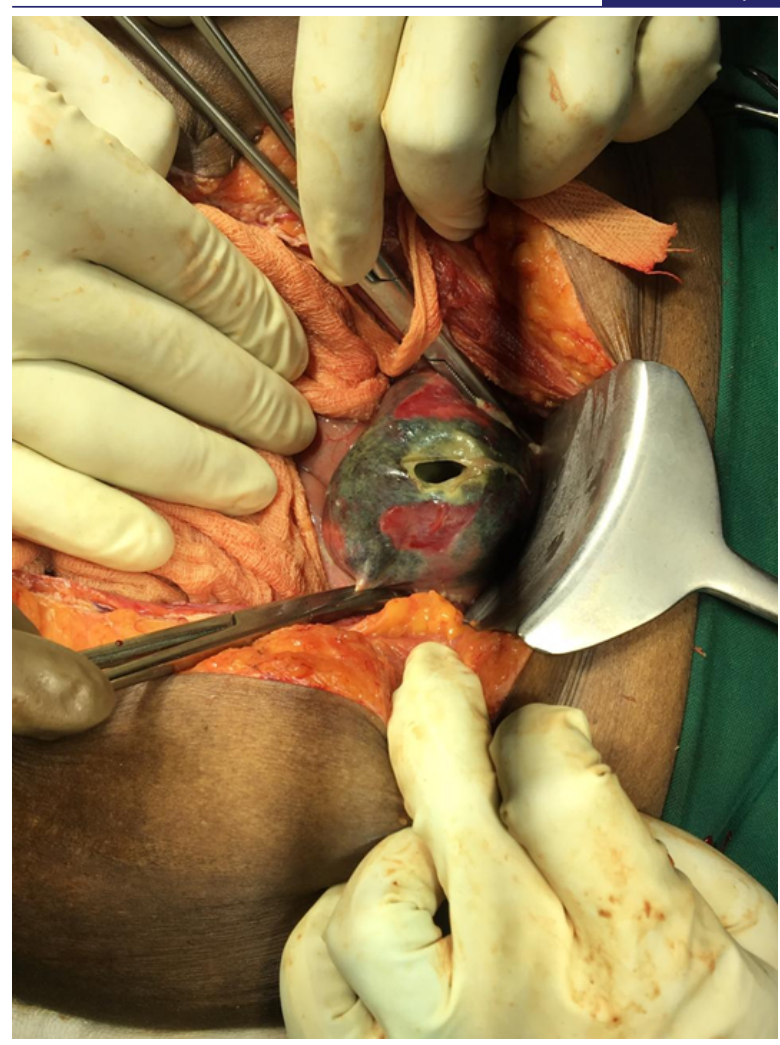

Intraoperative Laparotomy Picture Showing Large Perforation In Uterus.

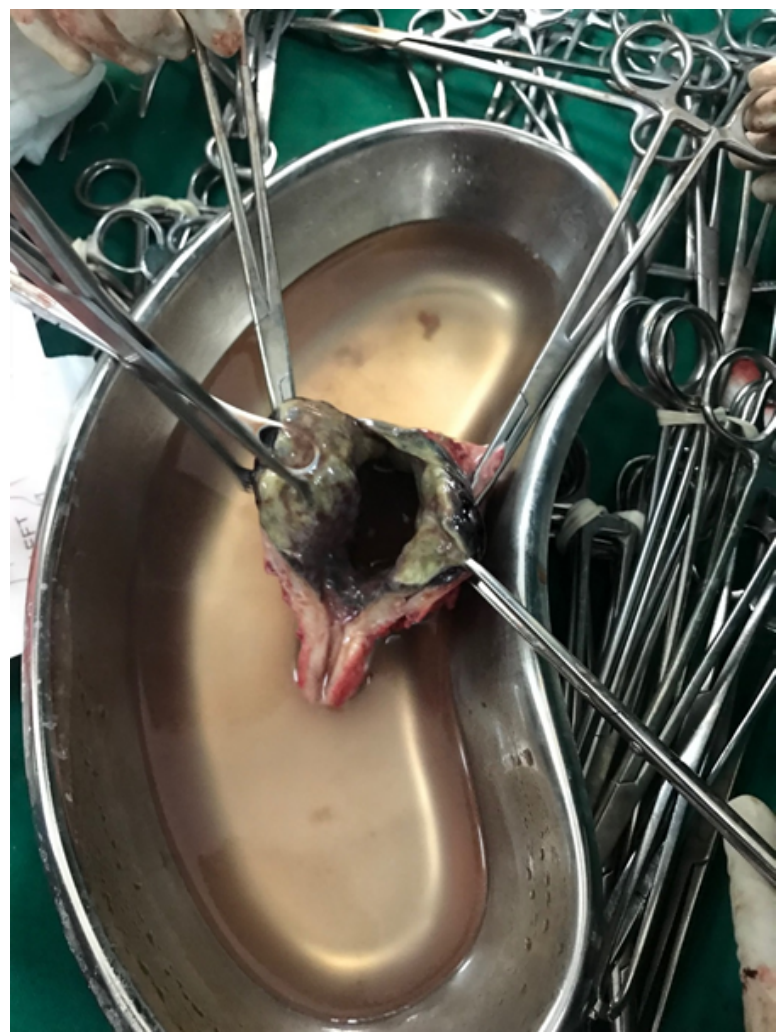

Postoperative Cut Surgical Specimen Showing Gangrenous Changes Of The Uterus.

\section{REFERENCES:}

1. David K, Nagarjuna M, Benjamin C, Fidenico D, Michael B, Akella C. Uterine perforation with gas gangrene

2. Kurashina A, Shimada H, Matsushima T, Doi D, Asakura H, Takeshita T. Spontaneous uterine perforation due to clostridial gas gangrene associated with endometrial carcinoma. J Nippon Med Sch. 2010;77:

3. Larson CM, Bubrick MP, Jacobs DM, West MA. Malignancy, mortality, and medicosurgical management of Clostridium septicum infection. Surgery 1995;118:592.

4. Nakamura M, Cross WR. The lecithinase (alpha toxin) activity of strains of Clostridium perfringens. Proc Soc Exp Biol Med 1968; 127:719. 